\title{
Waveforms for the Massive MIMO Downlink: Amplifier Efficiency, Distortion and Performance
}

\author{
Christopher Mollén, Erik G. Larsson and Thomas Eriksson
}

\section{Linköping University Post Print}

\section{Tweet}

N.B.: When citing this work, cite the original article.

(C2016 IEEE. Personal use of this material is permitted. However, permission to reprint/republish this material for advertising or promotional purposes or for creating new collective works for resale or redistribution to servers or lists, or to reuse any copyrighted component of this work in other works must be obtained from the IEEE.

Christopher Mollén, Erik G. Larsson and Thomas Eriksson, Waveforms for the Massive MIMO Downlink: Amplifier Efficiency, Distortion and Performance, 2016, IEEE Transactions on Communications, 99, 1-1.

http://dx.doi.org/10.1109/TCOMM.2016.2557781

Postprint available at: Linköping University Electronic Press

http://urn.kb.se/resolve?urn=urn:nbn:se:liu:diva-130514 


\title{
Waveforms for the Massive MIMO Downlink: Amplifier Efficiency, Distortion and Performance
}

\author{
Christopher Mollén, Erik G. Larsson and Thomas Eriksson
}

\begin{abstract}
In massive MIMO, most precoders result in downlink signals that suffer from high PAR, independently of modulation order and whether single-carrier or OFDM transmission is used. The high PAR lowers the power efficiency of the base station amplifiers. To increase power efficiency, low-PAR precoders have been proposed. In this article, we compare different transmission methods for massive MIMO in terms of the power consumed by the amplifiers. It is found that: 1) OFDM and single-carrier transmission have the same performance over a hardened massive MIMO channel and 2) when the higher amplifier power efficiency of low-PAR precoding is taken into account, conventional and low-PAR precoders lead to approximately the same power consumption. Since downlink signals with low PAR allow for simpler and cheaper hardware, than signals with high PAR, therefore, the results suggest that low-PAR precoding with either single-carrier or OFDM transmission should be used in a massive MIMO base station.
\end{abstract}

Index Terms-low-PAR precoding, massive MIMO, multiuser precoding, out-of-band radiation, peak-to-average ratio, power amplifier, power consumption.

\section{INTRODUCTION}

W IRELESS massive MIMO (Multiple-Input MultipleOutput) systems, initially conceived in [2] and popularly described in [3], simultaneously serve tens of users with base stations equipped with tens or hundreds of antennas using multiuser precoding. Compared to classical multiuser MIMO, order-of-magnitude improvements are obtained in spectral and energy efficiency [4], [5]. For these reasons, massive MIMO is expected to be a key component of future wireless communications infrastructure [3], [6].

This work compares different multiuser precoding techniques for the massive MIMO downlink. Under a total radiated power constraint, optimal multiuser MIMO precoding is a rather wellunderstood topic, see, e.g., [7], [8], as is linear (and necessarily suboptimal) precoding, see, e.g., [9] and the survey [10]. It is also known that, for massive MIMO specifically, linear precoding is close to optimal under a total-radiated power constraint [5]. There are also numerous results on precoding under per-antenna power constraints [11]-[13].

In practice, massive MIMO precoders optimized subject to a total radiated power constraint yield transmit signals with

C. Mollén and E. Larsson are with the Communication Systems Division, Dept. of Electrical Eng. (ISY), Linköping University, Linköping, Sweden, and T. Eriksson is with the Dept. of Signals and Systems, Chalmers University of Technology, Gothenburg, Sweden.

The research leading to these results has received funding from the European Union Seventh Framework Programme under grant agreement number ICT-619086 (MAMMOET), the Swedish Research Council (Vetenskapsrådet) and ELLIIT.

Parts of this work were presented at the European Wireless conference 2014 [1].

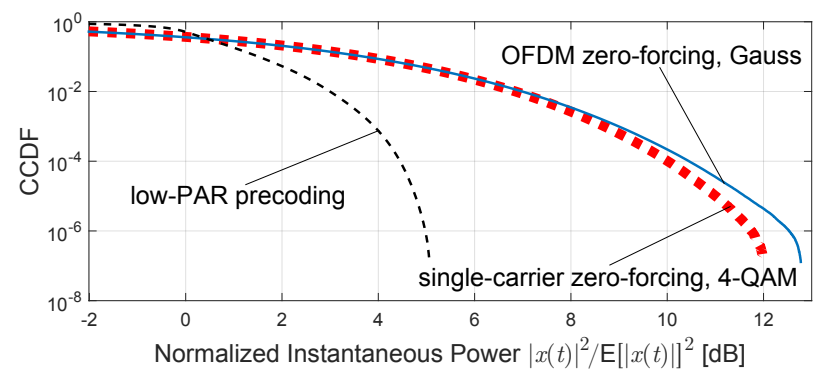

Fig. 1. The complimentary cumulative distribution of the amplitudes of different massive MIMO downlink signals that have been pulse shaped by a rootraised cosine filter, roll-off 0.22. Single-carrier and OFDM transmission have very similar distributions for the linear precoders described in Section III-B3 for any modulation order, cf. [15]. The low-PAR precoding technique is the one described in [16] and in Section III-C1. The system has 100 base station antennas, 10 single-antenna users and the channel is i.i.d. Rayleigh fading with 4 taps.

high peak-to-average ratio (PAR), regardless of whether singlecarrier or orthogonal frequency-division multiplexing (OFDM) transmission and of whether a low or a high modulation order is used, see Figure 1. To avoid heavy signal distortion and out-of-band radiation, transmission of such signals requires that the power amplifiers are backed off and operated at a point, where their transfer characteristics are sufficiently linear [14]. The higher the signal PAR is, the more backoff is needed; and the higher the backoff is, the lower the power efficiency of the amplifier will be. Against this background, precoders that yield signals with low PAR would be desirable.

The possibility to perform low-PAR precoding is a unique opportunity offered by the massive MIMO channel-signal peaks can be reduced because the massive MIMO downlink channel has a large nullspace and any additional signal transmitted in the nullspace does not affect what the users receive. In particular, PAR-reducing signals from the channel nullspace can be added to the downlink signal so that the emitted signals have low PAR [3], [17]. A few low-PAR precoders for massive MIMO have been proposed in the literature [16]-[18]. In [17], the discrete-time downlink signals were constrained to have constant envelopes. ${ }^{1}$ There it was estimated that, in typical massive MIMO scenarios, 1-2 dB extra radiated power is required to achieve the same sum-rate as without an envelope constraint. While some extra radiated power is required by low-PAR precoders, it was argued in [17]

\footnotetext{
${ }^{1}$ Note that the precoder in [17] can transmit symbols from any general input constellation and is compatible with both single-carrier transmission and OFDM - the received signals do not have to have constant envelopes, only the downlink signal emitted from each base station antenna has constant envelope.
} 
that the overall power consumption still should decrease due to the increased amplifier efficiency.

Another unique feature of the massive MIMO downlink channel is that certain types of hardware-induced distortion tend to average out when observed at the receivers [19]. Our study confirms that the variance of the in-band distortion caused by nonlinear base station amplifiers does decrease with the number of base station antennas.

Because the amount of power dissipation from the amplifiers depends on the amplitude distribution of the transmit signals, and because different transmit signals considered for massive MIMO systems have widely different amplitude distributions (widely different PAR), it is important to evaluate their performance in terms of the amount of power consumed by the amplifiers rather in terms of the amount of power that is radiated. The paper at hand compares precoders with different amplitude distributions in terms of amplifier power consumption and quantifies the benefits of low-PAR precoding for the massive MIMO downlink, taking into account in-band distortion and out-of-band radiation stemming from amplifier nonlinearities and imperfect channel state information due to pilot-based channel estimation. To the best knowledge of the authors, a comprehensive comparison, where amplifier efficiency and distortion are functions of the specific input signal rather than simplified functions of PAR, has not been done before. The difference between OFDM and single-carrier transmission is also investigated. The main technical contribution of the paper is a comprehensive end-to-end modeling of massive MIMO downlink transmission, which is treated in continuous time in order to capture the effects of nonlinear amplification, the associated capacity bound, and the estimations of the power consumption for relevant amplifier models. All conclusions are summarized in Section V.

We stress that the effect of amplifier nonlinearities on wireless signals have also been studied by others [14], [20], and for MIMO specifically in [21]. In relation to this literature, the novel aspects of our work include: (i) a specific focus on the massive MIMO downlink channel, which facilitates low-PAR precoding; (ii) a classification and comparison of precoders commonly considered for massive MIMO; (iii) an estimate of the amplifier power consumption of low-PAR precoding in comparison to that of other standard precoders.

\section{SYSTEM MODEL}

The downlink shown in Figure 2(a) is studied. The base station is equipped with $M$ antennas and it serves $K$ singleantenna users over a frequency-selective channel. All signals are modeled in complex baseband.

We let $s_{k}[n]$ be the $n$-th symbol that is to be transmitted to user $k$ and collectively denote all the $n$-th symbols by $\mathbf{s}[n] \triangleq$ $\left(s_{1}[n], \ldots, s_{K}[n]\right)$. The base station precodes the symbols to produce the discrete-time signals $\left\{u_{m}[n]\right\}$, where $u_{m}[n]$ is the precoded signal of antenna $m$. These signals are scaled such that

$$
\sum_{m=1}^{M} \mathrm{E}\left[\left|u_{m}[n]\right|^{2}\right]=1, \quad \forall n
$$

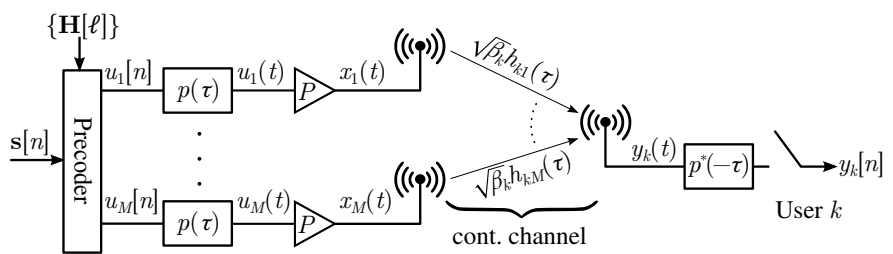

(a) The continuous-time model of the downlink.

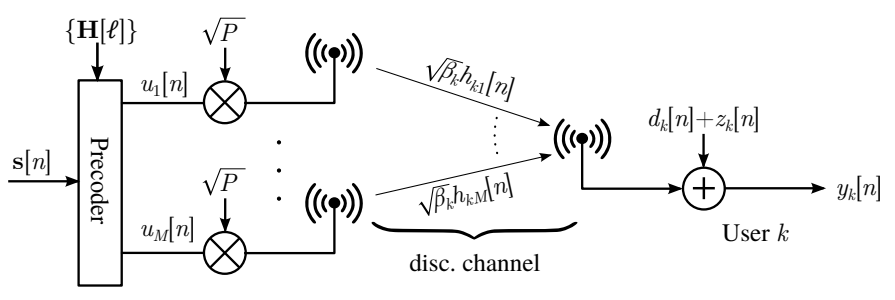

(b) The equivalent discrete-time model of the downlink.

Fig. 2. The downlink of a massive MIMO system.

and pulse shaped by a filter with impulse response $p(\tau)$ into the continuous-time transmit signals

$$
u_{m}(t) \triangleq \sum_{n} u_{m}[n] p(t-n T)
$$

where $T$ is the symbol period. After pulse shape filtering, the transmit signal $u_{m}(t)$ has a bandwidth smaller or equal to the bandwidth $B$ of the pulse $p(\tau)$. The bandwidth $B$ is the width of the interval, over which the spectrum of $p(\tau)$ is non-zero. For example, if a root-raised cosine filter of period $T$ with roll-off $\sigma$ were used, then $B T=1+\sigma$.

The continuous-time signal $u_{m}(t)$ is then amplified to transmit power by an amplifier that, in general, is nonlinear. The amplified signal is given by

$$
x_{m}(t)=g\left(\frac{\left|u_{m}(t)\right|}{\sqrt{b}}\right) e^{j\left(\arg u_{m}(t)+\Phi\left(\left|u_{m}(t)\right| / \sqrt{b}\right)\right)},
$$

where $g\left(\left|u_{m}(t)\right|\right)$ is the AM-AM conversion and $\Phi\left(\left|u_{m}(t)\right|\right)$ the AM-PM conversion ${ }^{2}$, see for example [15]. For now, the conversions $g(u)$ and $\Phi(u)$ are generic functions. Later in our analysis however, appropriate assumptions will be made to specify them. The factor $b$ is the backoff that has to be done to avoid nonlinear amplification and distortion. By backing off the signal power to a suitable operating point, the signal amplitude will stay in a region with sufficiently linear amplification most of the time, see [15]. In this article, all backoffs are given in $\mathrm{dB}$ relative to the backoff of the $1-\mathrm{dB}$ compression point-the point, where the output signal is $1 \mathrm{~dB}$ weaker than what it would have been if the amplification were perfectly linear. The signals are amplified so that

$$
\lim _{t_{0} \rightarrow \infty} \sum_{m=1}^{M} \mathrm{E}\left[\frac{1}{t_{0}} \int_{-t_{0} / 2}^{t_{0} / 2}\left|x_{m}(t)\right|^{2} \mathrm{~d} t\right]=P,
$$

where $P$ is the transmitted power of the base station.

The nonlinear relation in (3) generally widens the spectrum

${ }^{2}$ The acronyms in the terms AM-AM and AM-PM conversion stand for the fact that these functions describe how the modulation of the amplitude affects the Amplitude Modulation and Phase Modulation of the amplified signal. 


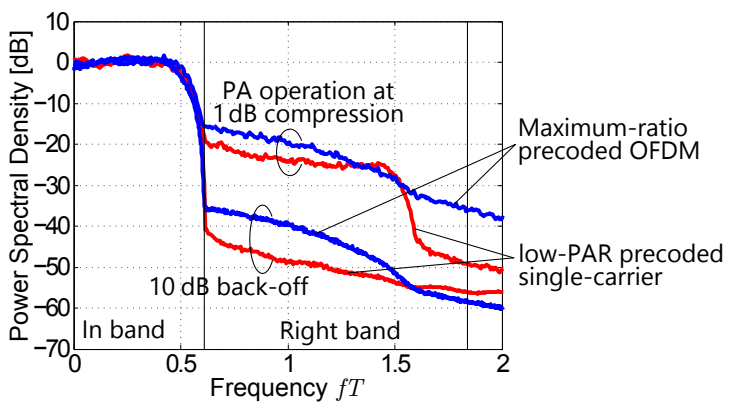

Fig. 3. The power spectral densities after amplification of two signal types with the PA operating at the $1 \mathrm{~dB}$ compression point (upper two curves) and well below saturation (lower two curves). The signals are from the system later described in Table I, where the bandwidth of the pulse $p(\tau)$ is $B T=1.22$.

of the amplified signal, i.e. the power of the signal is no longer confined to the bandwidth $B$ of the pulse $p(\tau)$. The power outside the ideal bandwidth is called out-of-band radiation and is quantified by the adjacent channel leakage ratio (ACLR), which is defined in terms of the power $P_{[-B / 2, B / 2]}$ of $x_{m}(t)$ in the useful band and the powers $P_{[-3 B / 2,-B / 2]}, P_{[B / 2,3 B / 2]}$ in the immediately adjacent bands:

$$
\mathrm{ACLR} \triangleq \max \left(\frac{P_{[-3 B / 2,-B / 2]}}{P_{[-B / 2, B / 2]}}, \frac{P_{[B / 2,3 B / 2]}}{P_{[-B / 2, B / 2]}}\right),
$$

where

$$
P_{\mathscr{B}} \triangleq \int_{f \in \mathscr{B}} S_{x}(f) \mathrm{d} f
$$

and $S_{x}(f)$ is the power spectral density of $x_{m}(t)$. In Figure 3, four power spectral densities of different amplified signals are shown to illustrate the out-of-band radiation. Half the in-band spectrum is shown together with the whole right band.

This signal is broadcast over the channel, whose small-scale fading impulse response from antenna $m$ to user $k$ is $h_{k m}(\tau)$ and large-scale fading coefficient to user $k$ is $\beta_{k}$. Specifically, user $k$ receives the signal

$$
y_{k}(t)=\sqrt{\beta_{k}} \sum_{m=1}^{M}\left(h_{k m}(\tau) \star x_{m}(\tau)\right)(t)+z_{k}(t),
$$

where $z_{k}(t)$ is a stationary white Gaussian stochastic process with spectral height $N_{0}$ that models the thermal noise of the user equipment. The received signal is passed through a filter matched to the pulse $p(\tau)$ and sampled to produce the discretetime received signal

$$
y_{k}[n] \triangleq\left(p^{*}(-\tau) \star y_{k}(\tau)\right)(n T) .
$$

In analyzing this system, we will look into an equivalent discrete-time system, see Figure 2(b). In order to do that, the distortion produced by the nonlinear amplifier has to be treated separately, since the nonlinearity widens the spectrum and is not accurately described by symbol-rate sampling. The smallscale fading coefficients of the discrete-time impulse response of the channel, including the pulse-shaping and matched filter, between antenna $m$ and user $k$ are denoted

$$
h_{k m}[\ell] \triangleq T\left(p(\tau) \star h_{k m}(\tau) \star p^{*}(-\tau)\right)(\ell T) .
$$

For these channel coefficients, we assume that

$$
\begin{aligned}
& \mathrm{E}\left[h_{k m}^{*}[\ell] h_{k m}\left[\ell^{\prime}\right]\right]=0, \quad \forall \ell \neq \ell^{\prime}, \\
& \sum_{\ell=0}^{L-1} \mathrm{E}\left[\left|h_{k m}[\ell]\right|^{2}\right]=1, \quad \forall k, m,
\end{aligned}
$$

and that $h_{k m}[\ell]$ is zero for integers $\ell \notin[0, L-1]$, where $L$ is the number of channel taps.

The $n$-th received sample at user $k$ is then given by

$$
y_{k}[n]=\sqrt{P \beta_{k}}\left(\sum_{m=1 \ell=0}^{M} \sum_{k-1}^{L-1} h_{k m}[\ell] u_{m}[n-\ell]+d_{k}[n]\right)+z_{k}[n],
$$

where the noise sample $z_{k}[n] \triangleq\left(p^{*}(-\tau) \star z_{k}(\tau)\right)(n T)$. To make $z_{k}[n] \sim \mathscr{C} \mathcal{N}\left(0, N_{0} / T\right)$ i.i.d., it is assumed that $p(\tau)$ is a root-Nyquist pulse of period $T$ and signal energy $1 / T$. The term $d_{k}[n]$ describes the in-band distortion - the part of the distortion that can be seen in the received samples $y_{k}[n]$ — caused by the nonlinear amplification of the transmit signal. It is given by $d_{k}[n] \triangleq$

$$
\frac{1}{\sqrt{P}} \sum_{m=1}^{M}\left(\left(x_{m}(t)-\sqrt{P} u_{m}(t)\right) \star h_{k m}(\tau) \star p^{*}(-\tau)\right)(n T) .
$$

By introducing the following vectors

$$
\begin{array}{ll}
\mathbf{u}[n] \triangleq\left(u_{1}[n], \ldots, u_{M}[n]\right)^{\top} & \mathbf{y}[n] \triangleq\left(y_{1}[n], \ldots, y_{K}[n]\right)^{\top} \\
\mathbf{d}[n] \triangleq\left(d_{1}[n], \ldots, d_{K}[n]\right)^{\top} & \mathbf{z}[n] \triangleq\left(z_{1}[n], \ldots, z_{K}[n]\right)^{\top}
\end{array}
$$

and matrices

$$
\mathbf{H}[\ell] \triangleq\left(\begin{array}{ccc}
h_{11}[\ell] & \cdots & h_{1 M}[\ell] \\
\vdots & \ddots & \vdots \\
h_{K 1}[\ell] & \cdots & h_{K M}[\ell]
\end{array}\right) \quad \mathbf{B} \triangleq \operatorname{diag}\left(\beta_{1}, \ldots, \beta_{K}\right),
$$

the received signals can be written as

$$
\mathbf{y}[n]=\sqrt{P} \mathbf{B}^{\frac{1}{2}}\left(\sum_{\ell=0}^{L-1} \mathbf{H}[\ell] \mathbf{u}[n-\ell]+\mathbf{d}[n]\right)+\mathbf{z}[n] .
$$

If the transmission were done in a block of $N$ symbols per user, and a cyclic prefix were used in front of the blocks, i.e.

$$
\mathbf{u}[n]=\mathbf{u}[N+n], \quad \text { for } n=-L+1, \ldots,-1,
$$

where $n=0$ is the time instant when the first symbol is received at the users, then the received signal in (15) is easily given in the frequency domain. If the discrete Fourier transforms of the transmit signals, received signals and channel are denoted by

$$
\begin{aligned}
& \tilde{\mathbf{u}}[\nu] \triangleq \sum_{n=0}^{N-1} e^{-j 2 \pi n \nu / N} \mathbf{u}[n], \\
& \tilde{\mathbf{y}}[\nu] \triangleq \sum_{n=0}^{N-1} e^{-j 2 \pi n \nu / N} \mathbf{y}[n], \\
& \tilde{\mathbf{H}}[\nu] \triangleq \sum_{\ell=0}^{L-1} e^{-j 2 \pi \ell \nu / N} \mathbf{H}[\ell],
\end{aligned}
$$

then the received signal at frequency index $\nu$ is given by

$$
\tilde{\mathbf{y}}[\nu]=\sqrt{P} \mathbf{B}^{\frac{1}{2}}(\tilde{\mathbf{H}}[\nu] \tilde{\mathbf{u}}[\nu]+\tilde{\mathbf{d}}[\nu])+\tilde{\mathbf{z}}[\nu],
$$


where $\tilde{\mathbf{d}}[\nu]$ describes the in-band distortion caused by the nonlinear amplification, $\tilde{\mathbf{z}}[\nu] \sim \mathscr{C} \mathcal{N}\left(0, \frac{N_{0}}{T} \mathbf{I}_{K}\right)$ and $\mathbf{I}_{K}$ is the $K$-dimensional identity matrix. The frequency-domain notation in (17)-(19) will be useful when we later consider OFDM-based transmission methods.

In this paper, we limit ourselves to look at block transmission with a cyclic prefix (16). To require a cyclic prefix simplifies our exposition and does not limit its generality much. A prefix is present in almost all modern digital transmission methods, as a guard interval or as a delimiter between blocks. A prefix that is correlated with the symbols is arguably a waste of spectral resources. However, by letting $N$ be much greater than $L$, this waste can be made arbitrarily small.

\section{DOWNLINK TRANSMISSION}

In the downlink, a precoder chooses, based on the channel state information available at the base station, transmit signals such that the users receive the symbols intended for them. The symbols to be transmitted fulfil

$$
\mathrm{E}\left[\left|s_{k}[n]\right|^{2}\right]=\xi_{k}, \quad \forall n, k,
$$

where $\left\{\xi_{k}\right\}$ are power allocation coefficients that are normalized such that

$$
\sum_{k=1}^{K} \xi_{k}=1
$$

We assume that the uplink and downlink are separated in time, using so called time-division duplexing, and that each user sends an $N_{\mathrm{p}}$-symbol long pilot sequence in the uplink that is orthogonal to the pilots of all other users. The pilots are used by the base station to estimate the small-scale fading coefficients $\left\{h_{k m}[\ell]\right\}$. The large scale fading coefficients $\left\{\beta_{k}\right\}$ are assumed to be known. Note that, to achieve orthogonality between pilots, $N_{\mathrm{p}} \geq K L$. Further, it is assumed that the channel estimates

$$
\hat{h}_{k m}[\ell]=h_{k m}[\ell]-\epsilon_{k m}[\ell], \quad \forall k, m, \ell,
$$

where $\epsilon_{k m}[\ell]$ is the estimation error, are obtained through linear minimum-mean-square estimation, so that $\hat{h}_{k m}[\ell]$ and $\epsilon_{k m}[\ell]$ are uncorrelated. In analogy with (19), we will denote the Fourier transforms of the channel estimates and the estimation error $\left\{\hat{\tilde{h}}_{k m}[\nu]\right\}$ and $\left\{\tilde{\epsilon}_{k m}[\nu]\right\}$ respectively. Their variances are

$$
\begin{aligned}
& \delta_{k} \triangleq \sum_{\ell=0}^{L-1} \mathrm{E}\left[\left|\hat{h}_{k m}[\ell]\right|^{2}\right]=\mathrm{E}\left[\left|\hat{\tilde{h}}_{k m}[\nu]\right|^{2}\right], \\
& \mathscr{E}_{k} \triangleq \sum_{\ell=0}^{L-1} \mathrm{E}\left[\left|\epsilon_{k m}[\ell]\right|^{2}\right]=\mathrm{E}\left[\left|\tilde{\epsilon}_{k m}[\nu]\right|^{2}\right] .
\end{aligned}
$$

Note that if $\left\{h_{k m}[\ell]\right\}$ are i.i.d. across $k$ and $m$ and if the uplink is perfectly linear, then

$$
\delta_{k}=\frac{N_{\mathrm{p}} \rho_{\mathrm{p}} \beta_{k}}{L+N_{\mathrm{p}} \rho_{\mathrm{p}} \beta_{k}}, \quad \mathscr{E}_{k}=\frac{L}{L+N_{\mathrm{p}} \rho_{\mathrm{p}} \beta_{k}},
$$

where $\rho_{\mathrm{p}}$ is the ratio between the power used to transmit the pilots and the thermal noise variance of a base station antenna.

\section{A. Achievable Data Rates}

In this section, an achievable rate is derived by breaking up the received signal into terms that are correlated to the desired signal $s_{k}[n]$ and terms that are uncorrelated to it. To treat single-carrier and OFDM transmission together, a common notation is introduced for the $n$-th received sample at user $k$, which is written as

$$
\bar{y}_{k}[n] \triangleq\left\{\begin{array}{ll}
y_{k}[n], & \text { if single-carrier transmission } \\
\tilde{y}_{k}[n], & \text { if OFDM transmission }
\end{array} .\right.
$$

This signal consists of three terms:

$$
\bar{y}_{k}[n]=\sqrt{P \beta_{k}}\left(r_{k}[n]+\bar{d}_{k}[n]\right)+\bar{z}_{k}[n],
$$

where

$$
r_{k}[n]= \begin{cases}\sum_{m=1}^{M} \sum_{\ell=0}^{L-1} h_{k m}[\ell] u_{m}[n-\ell], & \text { if SC } \\ \sum_{m=1}^{M} \tilde{h}_{k m}[n] \tilde{u}_{m}[n], & \text { if OFDM }\end{cases}
$$

is the linear part of the received signal and

$$
\bar{d}_{k}[n] \triangleq\left\{\begin{array} { l l } 
{ d _ { k } [ n ] } \\
{ \tilde { d } _ { k } [ n ] }
\end{array} \quad \overline { z } _ { k } [ n ] \triangleq \left\{\begin{array}{ll}
z_{k}[n], & \text { if SC } \\
\tilde{z}_{k}[n], & \text { if OFDM }
\end{array}\right.\right.
$$

are the in-band distortion and thermal noise terms.

By rewriting the channel in terms of the channel estimate and its error as in (23), the linear part can be further split up into

$$
r_{k}[n]=r_{k}^{\prime}[n]+e_{k}[n],
$$

where the useful signal and error due to imperfect channel state knowledge are

$$
\begin{aligned}
r_{k}^{\prime}[n] \triangleq \begin{cases}\sum_{m=1}^{M} \sum_{\ell=0}^{L-1} \hat{h}_{k m}[\ell] u_{m}[n-\ell], & \text { if SC } \\
\sum_{m=1}^{M} \hat{\tilde{h}}_{k m}[n] \tilde{u}_{m}[n], & \text { if OFDM }\end{cases} \\
e_{k}[n] \triangleq \begin{cases}\sum_{m=1}^{M} \sum_{\ell=0}^{L-1} \epsilon_{k m}[\ell] u_{m}[n-\ell], & \text { if SC } \\
\sum_{m=1}^{M} \tilde{\epsilon}_{k m}[n] \tilde{u}_{m}[n], & \text { if OFDM }\end{cases}
\end{aligned}
$$

It is observed that the error due to imperfect channel state knowledge at the base station is uncorrelated to the signal of interest: $\mathrm{E}\left[s_{k}^{*}[n] e_{k}[n]\right]=0$.

We have now two terms, $r_{k}^{\prime}[n]$ and $\bar{d}_{k}[n]$, that still might be correlated to the signal of interest $s_{k}[n]$. They can be divided into correlated and uncorrelated terms, as follows:

$$
\begin{aligned}
r_{k}^{\prime}[n] & =g_{k} \sqrt{\delta_{k}} s_{k}[n]+i_{k}[n] \\
\bar{d}_{k}[n] & =c_{k} \sqrt{\delta_{k}} s_{k}[n]+\rho_{k}\left(i_{k}[n]+e_{k}[n]\right)+d_{k}^{\prime}[n],
\end{aligned}
$$

where $i_{k}[n]$ and $d_{k}^{\prime}[n]$ are the residual interference terms that are uncorrelated with the symbol of interest, and with the sum $i_{k}[n]+e_{k}[n]$ in case of $d_{k}^{\prime}[n]$. The deterministic constants are given by

$$
\begin{aligned}
g_{k} & \triangleq \frac{1}{\sqrt{\delta_{k}} \xi_{k}} \mathrm{E}\left[s_{k}^{*}[n] r_{k}[n]\right], \\
c_{k} & \triangleq \frac{1}{\sqrt{\delta_{k}} \xi_{k}} \mathrm{E}\left[s_{k}^{*}[n] \bar{d}_{k}[n]\right], \\
\rho_{k} & \triangleq \frac{1}{I_{k}+E_{k}} \mathrm{E}\left[\left(i_{k}^{*}[n]+e_{k}^{*}[n]\right) \bar{d}_{k}[n]\right],
\end{aligned}
$$


where the channel error and interference variances are

$$
\begin{aligned}
E_{k} & \triangleq \mathrm{E}\left[\left|e_{k}[n]\right|^{2}\right], \\
I_{k} & \triangleq \mathrm{E}\left[\left|i_{k}[n]\right|^{2}\right] .
\end{aligned}
$$

We note that, when (26) holds, $E_{k}=\mathscr{E}_{k}$. The factors $g_{k}$ and $c_{k}$ are normalized by $\sqrt{\delta_{k}}$ so that they do not depend on the estimation error $\delta_{k}$. This normalization will later allow us to see the impact of the channel estimation error on the SINR. The fact that $g_{k}$ does not depend on $\delta_{k}$ is seen by expanding $g_{k}$ as is done in (84) in the Appendix. The interference $i_{k}[n]$ is uncorrelated with $s_{k}[n]$ because

$$
\begin{aligned}
\mathrm{E}\left[s_{k}^{*}[n] i_{k}[n]\right] & =\mathrm{E}\left[s_{k}^{*}[n]\left(r_{k}[n]-g_{k} \delta_{k}^{\frac{1}{2}} s_{k}[n]\right)\right] \\
& =\mathrm{E}\left[s_{k}^{*}[n] r_{k}[n]\right]-g_{k} \delta_{k}^{\frac{1}{2}} \xi_{k}=0 .
\end{aligned}
$$

That $d_{k}^{\prime}[n]$ is uncorrelated with both $s_{k}[n]$ and $i_{k}[n]+e_{k}[n]$ can be shown in the same way. The factor $c_{k}$ should be interpreted as the amount of amplitude that the nonlinear amplification "contributes" to the amplitude of the signal of interest. Usually, in a real-world system, this is a negative contribution in the sense that $\left|g_{k}+c_{k}\right|<\left|g_{k}\right|$. It should therefore be seen as the amount of amplitude lost (in what is usually called clipping). Similarly, the other correlation $\rho_{k}$ is the amount of interference that is clipped by the nonlinear amplification.

The whole received signal can thus be given as the sum of the following terms

$$
\begin{aligned}
\bar{y}_{k}[n]= & \sqrt{P \beta_{k}}\left(\sqrt{\delta_{k}}\left(g_{k}+c_{k}\right) s_{k}[n]\right. \\
& \left.+\left(1+\rho_{k}\right)\left(i_{k}[n]+e_{k}[n]\right)+d_{k}^{\prime}[n]\right)+\bar{z}_{k}[n]
\end{aligned}
$$

A lower bound ${ }^{3}$ on the capacity of the downlink channel to user $k$ is given by [22], [23]

$$
R_{k} \triangleq \log _{2}\left(1+\operatorname{SINR}_{k}\right) \text {, }
$$

where the signal-to-interference-and-noise ratio (SINR) is given by

$$
\operatorname{SINR}_{k}=\frac{\left|\mathrm{E}\left[\bar{y}_{k}^{*}[n] s_{k}[n]\right]\right|^{2} / \xi_{k}}{\mathrm{E}\left[\left|\bar{y}_{k}[n]\right|^{2}\right]-\left|\mathrm{E}\left[\bar{y}_{k}^{*}[n] s_{k}[n]\right]\right|^{2} / \xi_{k}} .
$$

Note that the capacity bound in (43) makes no assumption on Gaussianity of the interference terms. Further, it is not a function of the data symbols, only their second-order statistics.

To evaluate the expectations, we denote the variance of the in-band distortion

$$
D_{k} \triangleq \mathrm{E}\left[\left|d_{k}^{\prime}[n]\right|^{2}\right] .
$$

With this new notation, the two expectations in (44) can be written as follows.

$$
\begin{aligned}
\left|\mathrm{E}\left[\bar{y}_{k}^{*}[n] s_{k}[n]\right]\right|^{2} / \xi_{k}= & \delta_{k} \xi_{k} P \beta_{k}\left|g_{k}+c_{k}\right|^{2} \\
\mathrm{E}\left[\left|\bar{y}_{k}[n]\right|^{2}\right]= & P \beta_{k}\left(\delta_{k} \xi_{k}\left|g_{k}+c_{k}\right|^{2}\right. \\
& \left.+\left(I_{k}+E_{k}\right)\left|1+\rho_{k}\right|^{2}+D_{k}\right)+\frac{N_{0}}{T}
\end{aligned}
$$

\footnotetext{
${ }^{3}$ This analysis is general and does not make any assumption on Gaussianity of the transmit signals. In the computation of the capacity bound, however, a Gaussian distribution is used as one of the permissible distributions; its insertion into the expression for the mutual information yields a lower bound on capacity.
}

This simplifies (44), which becomes

$$
\operatorname{SINR}_{k}=\frac{\delta_{k} \xi_{k} P \beta_{k}\left|g_{k}+c_{k}\right|^{2}}{P \beta_{k}\left(\left(I_{k}+E_{k}\right)\left|1+\rho_{k}\right|^{2}+D_{k}\right)+\frac{N_{0}}{T}} .
$$

From (48), the two consequences of nonlinear amplification can be seen: (i) in-band distortion with variance $D_{k}$ and (ii) signal clipping by $c_{k}$, a reduction of the signal amplitude that results in a power-loss.

We also see that the variance $\delta_{k}$ is the fraction between the power that would have been received if the channel estimates were perfect and the actually received power. It can thus be seen as a measure of how much power that is lost due to imperfect channel state information at the base station.

The bound (43) is an achievable rate of a system that uses a given precoder and where the detector uses (36) as a channel estimate and treats the error terms in (42) as additional uncorrelated Gaussian noise. This detector has proven to be close to the optimal detector in environments, where the massive MIMO channel hardens.

\section{B. Linear Precoding Techniques}

With knowledge of the channel, the base station can precode the symbols in such a way that the gain $g_{k}$ is large and the interference $I_{k}$ small.

1) OFDM-Transmission: In OFDM transmission, the precoder is defined in the frequency domain. The time domain transmit signals are obtained from the inverse Fourier transform

$$
\mathbf{u}[n] \triangleq \sum_{\nu=0}^{N-1} e^{j 2 \pi n \nu / N} \tilde{\mathbf{u}}[\nu]
$$

of the precoded signals

$$
\tilde{\mathbf{u}}[\nu]=\tilde{\mathbf{W}}[\nu] \mathbf{s}[\nu], \quad \nu=1, \ldots, N-1
$$

where $\tilde{\mathbf{W}}[\nu]$ is a precoding matrix for frequency $\nu$. The precoding is linear, because the precoding matrix does not depend on the symbols, only on the channel.

To ensure that (1) is fulfilled, it is required that

$$
\mathrm{E}\left[\|\tilde{\mathbf{W}}[\nu]\|_{\mathrm{F}}^{2}\right]=K, \quad \forall \nu .
$$

2) Single-Carrier Transmission: The transmit signals of single-carrier transmission are given by the cyclic convolution

$$
\mathbf{u}[n]=\sum_{\ell=0}^{N-1} \mathbf{W}[\ell] \mathbf{s}[n-\ell],
$$

where the indices are taken modulo $N$. The impulse response of the precoder is given in terms of its frequency domain counterpart:

$$
\mathbf{W}[\ell] \triangleq \sum_{\nu=0}^{N-1} e^{j 2 \pi \nu \ell / N} \tilde{\mathbf{W}}[\nu] .
$$

3) Conventional Precoders: In this paper, three conventional precoders are studied. They will be given as functions of the channel estimates $\{\hat{\mathbf{H}}[\ell]\}$ (a sequence of matrices defined in 
terms of $\left\{\hat{h}_{k m}[\ell]\right\}$ in the same way as $\mathbf{H}[\ell]$ is defined in (14) in terms of $\left.\left\{h_{k m}[\ell]\right\}\right)$ and its Fourier transform

$$
\hat{\tilde{\mathbf{H}}}[\nu] \triangleq \sum_{\ell=0}^{N-1} \hat{\mathbf{H}}[\ell] e^{-j 2 \pi \nu \ell / N} .
$$

a) Maximum-Ratio Precoding: Maximum-ratio precoding is the precoder that maximizes the gain $g_{k}$ and the received power of the desired signal. It is given by

$$
\tilde{\mathbf{W}}[\nu]=\alpha_{\mathrm{MR}} \hat{\tilde{\mathbf{H}}}^{\mathrm{H}}[\nu], \quad \text { for } \mathrm{MR},
$$

where $\alpha_{\mathrm{MR}}$ is a power normalization factor that is chosen such that (51) holds. For maximum-ratio precoding

$$
\alpha_{\mathrm{MR}}^{2}=\frac{1}{M \sum_{k=1}^{K} \delta_{k}} .
$$

While it maximizes the received power of the transmission, interference $I_{k} \neq 0$ is still present in the received signal. In typical scenarios with favorable propagation, maximum-ratio precoding suppresses this interference increasingly well with higher number of base station antennas and in the limit of infinitely many antennas, the interference becomes negligible in comparison to the received power [2]. For maximum-ratio precoding and an i.i.d. Rayleigh fading channel, both with single-carrier and OFDM transmission, the array gain and interference terms are [5]

$$
g_{k}=\sqrt{M}, \quad I_{k}=\delta_{k}, \quad \text { for MR. }
$$

Because the precoding weights of antenna $m$ only depend on the channel coefficients $\left\{\hat{h}_{k m}[\ell]\right\}$ of that antenna, maximumratio precoding can be implemented in a distributed fashion, where the precoding is done locally at each antenna.

Note that this definition of maximum-ratio precoding makes it equivalent to time-reversal precoding for single-carrier transmission, see for example [24].

b) Zero-Forcing Precoding: The zero-forcing precoder is given by

$$
\tilde{\mathbf{W}}[\nu]=\alpha_{\mathrm{ZF}} \hat{\tilde{\mathbf{H}}}^{\mathrm{H}}[\nu]\left(\hat{\tilde{\mathbf{H}}}[\nu] \hat{\tilde{\mathbf{H}}}^{\mathrm{H}}[\nu]\right)^{-1} \quad \text { for } \mathrm{ZF},
$$

where $\alpha_{\mathrm{ZF}}$ is a power normalization factor that is chosen such that (51) holds. For zero-forcing precoding

$$
\alpha_{\mathrm{ZF}}=\frac{M-K}{\sum_{k=1}^{K} \frac{1}{\delta_{k}}},
$$

in the derivation of which we used the fact [25] that

$$
\begin{aligned}
\mathrm{E}\left[\left\|\tilde{\mathbf{H}}^{\mathrm{H}}[\nu]\left(\tilde{\mathbf{H}}[\nu] \tilde{\mathbf{H}}^{\mathrm{H}}[\nu]\right)^{-1}\right\|^{2}\right] & =\mathrm{E}\left[\operatorname{tr}\left(\tilde{\mathbf{H}}[\nu] \tilde{\mathbf{H}}^{\mathrm{H}}[\nu]\right)^{-1}\right] \\
& =\frac{K}{M-K} .
\end{aligned}
$$

The zero-forcing precoder nulls the interference $I_{k}$ at the cost of a lower gain $g_{k}$ compared to maximum-ratio precoding. For zero-forcing precoding and an i.i.d. Rayleigh fading channel, both with single-carrier and OFDM transmission, the gain and interference terms are [5]

$$
g_{k}=\sqrt{M-K}, \quad I_{k}=0, \quad \text { for } \mathrm{ZF} .
$$

c) Regularized Zero-Forcing Precoding: Regularized zeroforcing precoding aims at maximizing the received SINR (48). In the limit of an infinite number of antennas, the optimal linear precoder is given by [26]

$$
\tilde{\mathbf{W}}[\nu]=\alpha_{\mathrm{RZF}} \hat{\tilde{\mathbf{H}}}^{\mathrm{H}}[\nu]\left(\hat{\tilde{\mathbf{H}}}[\nu] \hat{\tilde{\mathbf{H}}}^{\mathrm{H}}[\nu]+\rho \mathbf{I}_{K}\right)^{-1}, \quad \text { for RZF, }
$$

where $\alpha_{\mathrm{RZF}}$ is a power normalization factor that is chosen such that (51) holds. For regularized zero-forcing, the factor $\alpha_{\mathrm{RZF}}$ is not known in closed form but it can easily be determined numerically as

$$
\alpha_{\mathrm{RZF}}^{2}=\frac{K}{\mathrm{E}\left[\left\|\hat{\tilde{\mathbf{H}}}^{\mathrm{H}}[\nu]\left(\hat{\tilde{\mathbf{H}}}[\nu] \hat{\tilde{\mathbf{H}}}^{\mathrm{H}}[\nu]+\rho \mathbf{I}_{K}\right)^{-1}\right\|^{2}\right]} .
$$

The factor $\rho \in \mathbb{R}^{+}$is a system parameter, which depends on the ratio $P T / N_{0}$ and on the path losses $\left\{\beta_{k}\right\}$ of the users. The regularized zero-forcing precoder balances the interference suppression of zero-forcing and array gain of maximum-ratio precoding [10] by changing the parameter $\rho$. How to find the optimal parameter $\rho$ is described in [26] and later in Section IV-B.

The interference $I_{k}$ and gain $g_{k}$ of regularized zero-forcing depend on the parameter $\rho$ and no closed-form expression for them is known. However, when the transmit power $P$ is low compared to the noise variance $N_{0} / T$, then a large $\rho$ is optimal and the interference and array gain are close to the ones of maximum-ratio precoding. And when the transmit power relative the noise variance is high, a small $\rho$ is optimal and the interference and array gain are close to the ones of zero-forcing.

\section{Low-PAR Precoding Techniques}

A low-PAR precoder produces transmit signals whose envelope varies little above the average envelope amplitude, i.e. signals with low PAR. Generally, the cost of the lowering PAR is a reduced gain $g_{k}$ or an increased interference $I_{k}$ as compared to conventional precoding techniques, where the envelope of the transmit signals is not constrained. We study two low-PAR precoders: the discrete-time constant-envelope precoder, originally proposed in [27] and extended in [16], [17], and the PAR-aware precoder proposed in [18].

1) Discrete-Time Constant-Envelope Precoding: The discrete-time constant-envelope precoder produces transmit signals that have constant-envelope when viewed in discrete time, i.e.

$$
\left|u_{m}[n]\right|=\frac{1}{\sqrt{M}}, \quad \forall n, m .
$$

It does so by minimizing the difference between the received noise-free signal and the desired symbols under a fixed modulus constraint. Given any symbol vector $\mathbf{s}[n]$, the single-carrier transmit signal $\left\{u_{m}[n]\right\}$ is obtained as the solution to:

$$
\underset{\left\{\left|u_{m}[n]\right|=M^{-1 / 2}\right\}}{\arg \min } \sum_{n=0}^{N-1}\left\|\sum_{\ell=0}^{L-1} \hat{\mathbf{H}}[\ell] \mathbf{u}[n-\ell]-\sqrt{\gamma} \mathbf{s}[n]\right\|^{2},
$$

where $\gamma \in \mathbb{R}^{+}$is a system parameter that is chosen to maximize the system performance. Intuitively, a small $\gamma$ makes the 
interference $I_{k}$ small, but the gain $g_{k}$ small too. On the other hand, a large $\gamma$ makes the gain large but also makes it hard to produce the desired symbol at each user, which results in an excessive amount of interference. In Section IV-B, it will be shown how the parameter $\gamma$ is chosen such that the data rate is maximized.

The optimization problem in (64) can be approximately solved at a low computational complexity by cyclic optimization: minimizing the norm with respect to one $u_{m}[n]$ at a time, while keeping the other variables fixed. Such a solver is not much heavier in terms of computations than the zero-forcing precoder [16].

2) PAR-Aware Precoding [18]: The PAR-aware precoder offers the possibility to balance the PAR reduction and the degradation of gain and interference variance. Given any symbol vector $\mathbf{s}[n]$, the single-carrier transmit signals are given as the solution to the following optimization problem

$$
\begin{aligned}
\left\{\mathbf{u}^{\prime}[n]\right\} \triangleq \underset{\left\{\mathbf{u}^{\prime}[n]\right\}}{\arg \min } & \left(\lambda \max \left\{\left\|\mathbf{u}^{\prime}[n]\right\|_{\infty}\right\}\right. \\
& \left.+\sum_{n=0}^{N-1}\left\|\sum_{\ell=0}^{L-1} \hat{\mathbf{H}}[\ell] \mathbf{u}[n-\ell]-\mathbf{s}[n]\right\|^{2}\right),
\end{aligned}
$$

which is normalized to unit power to give the transmit signals

$$
\mathbf{u}[n]=\frac{\mathbf{u}^{\prime}[n]}{\sqrt{\mathrm{E}\left[\left\|\mathbf{u}^{\prime}[n]\right\|^{2}\right]}} .
$$

The regularizing parameter $\lambda$ is used to control how much the PAR shall be reduced by penalizing the transmit signal with the largest envelope. An efficient solver to the optimization problem in (65) is presented in [18] for the case, in which the transmit signal has a cyclic prefix.

3) OFDM Transmission: OFDM transmission in connection with discrete-time constant-envelope precoding and PAR-aware precoding can be done with the same algorithms as for singlecarrier transmission. Instead of precoding the symbols $\{\mathbf{s}[n]\}$ directly, the base station would precode their inverse Fourier transform

$$
\tilde{\mathbf{s}}[n] \triangleq \frac{1}{\sqrt{N}} \sum_{\nu=0}^{N-1} e^{j 2 \pi \nu n / N} \mathbf{s}[\nu] .
$$

The convolutions in (64) and (65) should then be seen as cyclic, i.e. the indices should be taken modulo $N$.

\section{Power Allocation among Users}

The power allocation $\left\{\xi_{k}\right\}$ between users has to be decided according to a chosen criterion, for example that all users shall be served with the same data rate. This "egalitarian" criterion is used in this paper and is given by the max-min problem:

$$
\left\{\xi_{k}\right\}=\underset{\left\{\xi_{k}\right\}: \text { eq.(22) }}{\arg \max } \min \operatorname{SINR}_{k},
$$

where $\operatorname{SINR}_{k}$ is given in (48). Note that out of all the terms in (48), apart from $\xi_{k}$ itself, only the clipping $c_{k}$, in-band distortion $D_{k}$ and the correlation $\rho_{k}$ might depend on $\xi_{k}$. That $g_{k}$ and $I_{k}$ do not depend on $\left\{\xi_{k}\right\}$, can be seen from (86) and (91) in the Appendix. Extensive simulations over Rayleigh fading channels indicate that only $D_{k}$ depends on the power allocation $\xi_{k}$ and that this dependence is linear.

To solve (68), a first-order approximation of the dependence on $\xi_{k}$ is made. The in-band distortion is assumed to be:

$$
D_{k}=D^{\prime}+\delta_{k} \xi_{k} D^{\prime \prime},
$$

where $D^{\prime}$ and $D^{\prime \prime}$ are non-negative constants. This corresponds to assuming that the in-band distortion consists of two parts One part $D^{\prime}$ that is radiated isotropically from the base station and one part $D^{\prime \prime}$ that is beamformed in the same way as the useful signal. To compute the coefficients $D^{\prime}$ and $D^{\prime \prime}$, a Monte Carlo simulation was used, where the in-band distortion was measured for different values of $\delta_{k} \xi_{k}$. The coefficients $D^{\prime}$ and $D^{\prime \prime}$ were chosen as the least-squares solution to the fitting of the data to the model. To give a measure of the accuracy of the model, the relative mean-square-error was computed:

$$
\text { RELMSE }=\frac{\sum_{i}\left(D_{i}-\left(D^{\prime}+\delta_{i} \xi_{i} D^{\prime \prime}\right)\right)^{2}}{\sum_{i} D_{i}^{2}},
$$

where the index $i$ runs over all samples that were randomly generated. For example, in the simulation of a single-carrier system with 100 antennas using maximum-ratio precoding and a 4-tap channel, this normalized mean-square-error was smaller than $0.55 \%$ for all backoffs for 10 users and smaller than $0.03 \%$ for 50 users. The error of the modeled distortion variance is thus small compared to the magnitude of the actual distortion variance.

For the $\left\{\xi_{k}\right\}$ that solve (68), there is a common SINR such that $\operatorname{SINR}_{k}=\mathrm{SINR}$, for all $k$, because (48) is an increasing function in $\xi_{k}$. Rearranging (48) gives the power allocation

$$
\xi_{k}=\operatorname{SINR} \frac{P \beta_{k}\left(\left(I_{k}+E_{k}\right)\left|1+\rho_{k}\right|^{2}+D^{\prime}\right)+\frac{N_{0}}{T}}{\delta_{k} P \beta_{k}\left(\left|g_{k}+c_{k}\right|^{2}-D^{\prime \prime} \text { SINR }\right)} .
$$

Because the power allocations sum to one (22),

$$
\operatorname{SINR} \sum_{k=1}^{K} \frac{P \beta_{k}\left(\left(I_{k}+E_{k}\right)\left|1+\rho_{k}\right|^{2}+D^{\prime}\right)+\frac{N_{0}}{T}}{\delta_{k} P \beta_{k}\left(\left|g_{k}+c_{k}\right|^{2}-D^{\prime \prime} \operatorname{SINR}\right)}=1,
$$

the common SINR can be found by solving this equation. The optimal power allocations are thus given by (71), where SINR is the largest solution to (72).

Note that, if $D^{\prime \prime}=0,(72)$ can be solved explicitly, which gives an expression for SINR and the optimal power allocation

$$
\xi_{k}=\frac{\frac{1}{\delta_{k} \beta_{k}\left|g_{k}+c_{k}\right|^{2}}\left(P \beta_{k}\left(\left(I_{k}+E_{k}\right)\left|1+\rho_{k}\right|^{2}+D^{\prime}\right)+N_{0} / T\right)}{\sum_{k^{\prime}=1}^{K} \frac{P \beta_{k^{\prime}}\left(\left(I_{k^{\prime}}+E_{k^{\prime}}\right)\left|1+\rho_{k^{\prime}}\right|^{2}+D^{\prime}\right)+N_{0} / T}{\delta_{k^{\prime}} \beta_{k^{\prime}}\left|g_{k^{\prime}}+c_{k^{\prime}}\right|^{2}}} .
$$

When (26) holds, $\delta_{k}+E_{k}=1$. Therefore for maximum-ratio precoding, this power allocation becomes

$$
\xi_{k}^{\mathrm{MR}}=\frac{P \beta_{k}\left(\left|1+\rho_{k}\right|^{2}+D^{\prime}\right)+N_{0} / T}{\beta_{k} \delta_{k} \sum_{k^{\prime}=1}^{K} \frac{P \beta_{k^{\prime}}\left(\left|1+\rho_{k^{\prime}}\right|^{2}+D^{\prime}\right)+N_{0} / T}{\beta_{k^{\prime}} \delta_{k^{\prime}}}}, \quad \forall k,
$$

and, for zero-forcing precoding, it becomes

$$
\xi_{k}^{\mathrm{ZF}}=\frac{P \beta_{k}\left(E_{k}\left|1+\rho_{k}\right|^{2}+D^{\prime}\right)+N_{0} / T}{\beta_{k} \delta_{k} \sum_{k^{\prime}=1}^{K} \frac{P \beta_{k^{\prime}}\left(E_{k^{\prime}}\left|1+\rho_{k^{\prime}}\right|^{2}+D^{\prime}\right)+N_{0} / T}{\beta_{k^{\prime}} \delta_{k^{\prime}}}}, \quad \forall k .
$$

These two expressions (74) and (75) are equivalent to the 


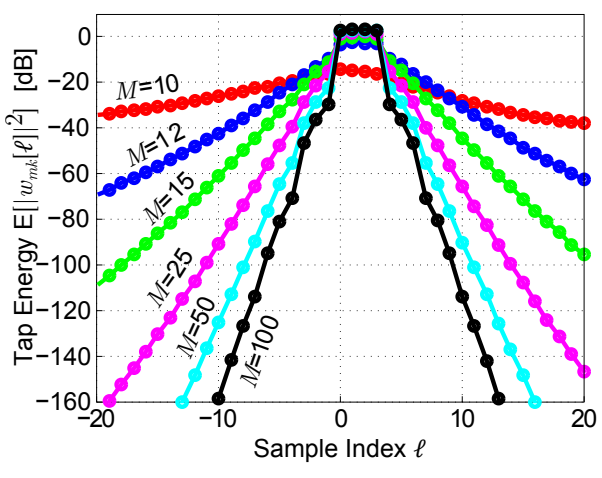

Fig. 4. The normalized energy of the taps of the impulse response of the zero-forcing filter for single-carrier transmission over a frequency-selective 4-tap channel. The base station serves $K=10$ users.

corresponding formulas in [28] in the special case there is no amplifier distortion. It should be noted that when the number of users is large, $K \gtrsim 30$, the term $D^{\prime \prime}$ is close to zero.

\section{E. Single-Carrier vs. OFDM Transmission}

The performance of single-carrier and OFDM transmission is the same in terms of the achievable rate (43), which has been proven tight when the channel hardens. Due to channel hardening, all tones have equally good channels $\{\tilde{\mathbf{H}}[n]\}$, therefore the advantage of OFDM-the possibility to do waterfilling across frequency-results in little gain independently of the transmit power $\frac{P T}{N_{0}}$. This is summarized in the following Proposition and proven in the Appendix.

Proposition 1: For any precoding technique $f: \tilde{\mathbf{H}}[n] \mapsto$ $\tilde{\mathbf{W}}[n], \forall n$, the rate in (43) is equal for single-carrier transmission (52) and for OFDM (49).

The computational complexity of the two transmission methods is also the same. In OFDM however, one of the Fourier transforms (53) is performed by the users instead of the base station, which performs the transform in single-carrier transmission.

While OFDM causes a delay of at least $N$ symbols, since precoding and detection are done block by block, singlecarrier transmission can be implemented for frequency-selective channels with short filters with delays in the order of $\sim L \ll N$. Channel inversion with filters with few taps is only possible in massive MIMO-in single-input single-output systems or small MIMO systems, pre-equalization of a frequency-selective channel requires filters with a large number of taps. This can be seen in Figure 4, which shows the power profile $\left\{\mathrm{E}\left[\left|w_{m k}[\ell]\right|^{2}\right], \ell=\ldots,-1,0,1, \ldots\right\}$ of the impulse response of the zero-forcing precoder for different numbers of base station antennas. It is seen that zero-forcing requires many filter taps when the number of antennas is small, while it requires approximately the same number of filter taps as the number of channel taps $L$ in massive MIMO, where the number of antennas is large.

An OFDM system is less sensitive to a time synchronization error in the sampling timing in (8) than a single-carrier system, because the symbol period of OFDM is longer than that of single-carrier transmission, $N T$ compared to $T$. A small time synchronization error, in the order of $T$, leads to a simple phase
TABLE I

SYSTEM PARAMETERS

number of tx-antennas $\quad M=100$

number of users

$K=10$ and 50

channel model

$L=4$-tap Rayleigh fading

pulse shape filter

amplifier type

path loss exponent

root-raised cosine, roll-off 0.22

class $\mathrm{B}$, see (80) and (77)

$\alpha=3.8$ (typical urban scenario)

rotation of the symbol estimates in OFDM, while it leads to difficult intersymbol interference in single-carrier transmission. On the other hand, a single-carrier system is less sensitive to a frequency synchronization error than an OFDM system. A small frequency synchronization error, in the order $\frac{1}{N T}$, leads to severe intersymbol interference in OFDM, while it only leads to a simple phase rotation in single-carrier transmission.

We stress that the signals of single-carrier and OFDM transmission practically have the same PAR in massive MIMO, at least in an i.i.d. Rayleigh fading environment. In Figure 1, there was only a small gap between the PAR of single-carrier and OFDM transmission and, when the number of channel taps is greater than $L>4$, the gap is practically closed. Neither of the transmission methods is therefore better than the other with respect to amplifier performance.

The operational differences between single-carrier and OFDM transmission are summarized in Table II.

\section{Numerical Evaluations of Rate}

To estimate the power consumed by the amplifiers at different sum-rates and to compare different precoders to each other, the expectations in (43) that lack closed-form expressions were numerically evaluated for the system specified in Table I. All continuous signals were modeled by $\kappa=7$ times oversampled discrete-time signals. Specifically the channel from antenna $m$ to user $k$ was assumed to be Rayleigh fading $h_{k m}(\ell T / \kappa) \sim$ $\mathscr{C} \mathcal{N}(0,1 /(\kappa L))$, for $\ell=0, \ldots, \kappa(L-1)$ and i.i.d. across $k$, $m$ and $\ell$. The users were assumed to be uniformly spread out over an annulus-shaped area, with inner radius $r$ and outer radius $100 r$. The path loss of user $k$ was then assumed to be

$$
\beta_{k}=\left(r / d_{k}\right)^{\alpha},
$$

where $d_{k}$ is the distance between user $k$ and the base station, which is located in the middle of the annulus, and where $\alpha$ is the path loss exponent.

Further, it was assumed that the pilots used for channel estimation were $N_{\mathrm{p}}=K L$ symbols long and sent with the same power $\rho_{\mathrm{p}}$ from all users. The power was chosen such that a signal sent from the cell edge, where the path loss is $\beta_{\min }=1 / 100^{\alpha}$, would be received at the base station with power $0 \mathrm{~dB}$ above the noise variance, i.e. $\rho_{\mathrm{p}} \beta_{\min }=N_{0} / T$ and $\rho_{\mathrm{p}}=100^{\alpha} N_{0} / T$.

\section{A. Effects of Nonlinear Power Amplifiers}

The power amplifiers of the simulated system have been modeled by the Rapp model [15], where the phase distortion 


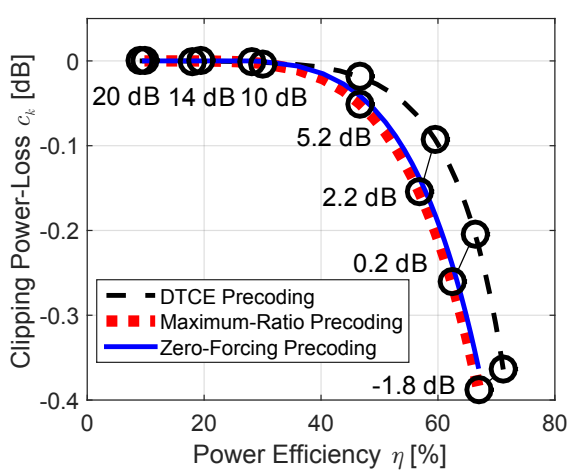

(a) The fraction of power lost due to clipping

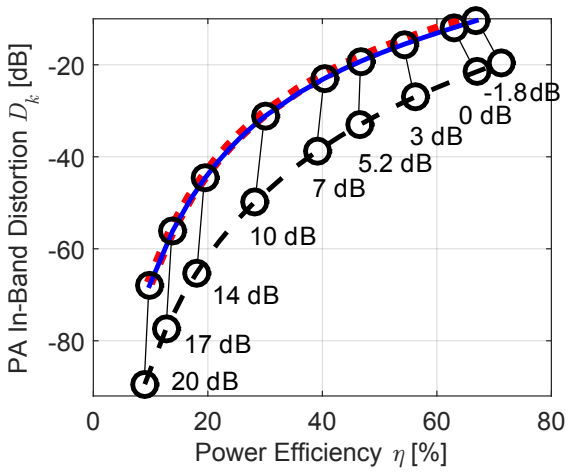

(b) The variance of the received in-band distortion

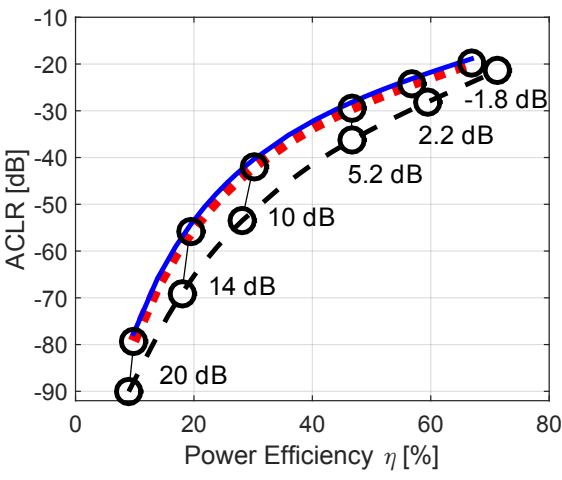

(c) The amount of out-of-band radiation

Fig. 5. Measurements on a Rapp-modeled $(p=2)$ class B amplifier for three signal types. The signals have been pulse shape filtered with a root-raised cosine, roll-off factor 0.22 . The encircled points correspond to some selected operating points of the amplifier, which are specified by the backoff from the 1 -dB compression point. It is assumed that all users are at the same distance to the base station, i.e. $\xi_{k}=1 / K$, for all $k$.

is neglected, so $\Phi(u)=0, \forall u$, and the AM-AM conversion is given by

$$
g(u)=A_{\max } \frac{u / u_{\max }}{\left(1+\left(u / u_{\max }\right)^{2 p}\right)^{\frac{1}{2 p}}},
$$

where the parameter $p=2$ approximates a typical moderatecost solid-state power amplifier [29]. The parameter $A_{\max }$ is the highest possible output amplitude and $u_{\max }=A_{\max } / g^{\prime}(0)$ determines the slope of the asymptote that $g(u)$ approaches for small $u$.

To ensure that the total radiated power is $P$, as is required by (4), the parameters are chosen as follows:

$$
\begin{aligned}
u_{\max } & =M^{-1 / 2} \\
A_{\max } & =u_{\max } \frac{\sqrt{P}}{\lambda_{0}},
\end{aligned}
$$

where $\lambda_{0}$ is a correction factor to compensate for the power lost due to clipping, which is chosen such that (4) holds. Note that the correction factor is different for different signal types and backoffs.

Massive MIMO will require simple, inexpensive and power efficient amplifiers [3]. The most basic class B amplifiers have these properties [30], and could therefore potentially be suited for massive MIMO. The power efficiency of such an amplifier is given by [15]

$$
\eta=\frac{\pi}{4} \frac{\mathrm{E}\left[g^{2}\left(\left|u_{m}(t)\right|\right)\right]}{A_{\max } \mathrm{E}\left[g\left(\left|u_{m}(t)\right|\right)\right]},
$$

Note that $\eta \leq \pi / 4$, with equality only if the continuous-time input signal $u_{m}(t)$ has perfectly constant envelope and the amplifier operates in saturation.

The two phenomena of nonlinear amplification-in-band distortion and amplitude clipping — are studied by looking at the variance of the in-band distortion $\sigma_{k}^{2} \triangleq \frac{D_{k}}{\xi_{k}\left|g_{k}\right|^{2}}$ and the clipped power $a_{k} \triangleq \frac{\left|g_{k}+c_{k}\right|^{2}}{\left|g_{k}\right|^{2}}$ relative to the ideal amplitude.

The clipped power was computed together with the power efficiency of the amplifiers for several backoffs and averaged over many channel realizations for the system specified in Table I, in which all users are at the same distance to the base station and $\xi_{k}=1 / K$, for all $k$. By treating the backoff as an intermediate variable, the clipped power can be given as a function of the efficiency, see Figure 5(a). It is noted that the power lost due to clipping is small (smaller than $-0.4 \mathrm{~dB}$ ) even when the amplifiers are operated close to saturation.

Similarly, the variance of the in-band distortion, Figure 5(b), and the ACLR, Figure 5(c), were computed for several backoffs and averaged over different channel realizations. It can be seen that the amount of power radiated out-of-band is monotonically decreasing with the backoff. A constraint on the ACLR will therefore constrain the maximum efficiency that the amplifier can operate at. Further, it is noted that the efficiency is not a simple function of the backoff, but it depends on the signal type. We also note that, whereas the clipping power-loss is small at operating points with high efficiency, the in-band distortion (at least for the conventional precoders) and the out-of-band radiation are not. The latter two phenomena will thus be the main factors to determine the operating point of the amplifiers.

Because of their similar amplitude distributions, all the linear precoding techniques (maximum-ratio, zero-forcing and regularized zero-forcing precoding) result in similar curves in Figures 5(a), 5(b) and 5(c). Therefore, only the results of singlecarrier maximum-ratio and zero-forcing precoding are shown. The curves are identical to the ones of OFDM transmission.

In Figure 6, it can be seen how the effects of the nonlinear amplifiers change when the number of antennas, users and channel taps are changed in a single-carrier MIMO system. To make comparisons easy, all users in the system of Figure 6 have the same path loss and the amplifiers are backed off by $1 \mathrm{~dB}$, enough to see distinct clusters around each symbol point in all cases.

When the number of users and channel taps are small, the distribution of the in-band distortion is different around different symbol points and the phase tends to be more accurately reproduced than the amplitude, resulting in oblong clouds around the outer symbol points. It is a well-known phenomenon in OFDM with a great number of subcarriers that the distribution of the in-band distortion is almost circularly symmetric Gaussian and identically distributed around each symbol point, which means that the distortion can be regarded as uncorrelated additive noise [20]. In multiuser MIMO, a similar 

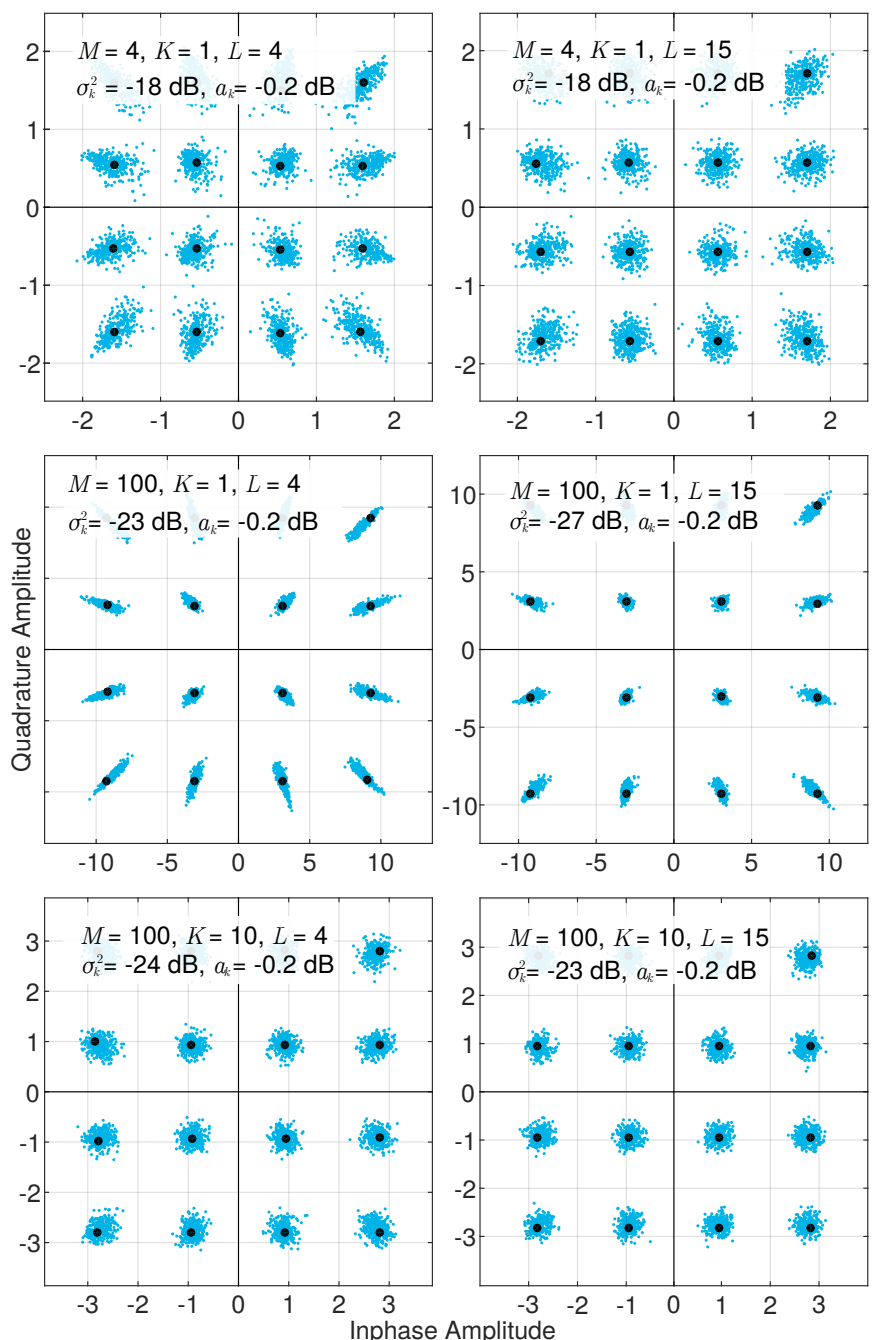

Fig. 6. Received signal points without thermal noise when broadcasting 16-QAM symbols with nonlinear amplification ( $1 \mathrm{~dB}$ backoff) over a MIMO channel by single-carrier zero-forcing precoding for different number of users $K$, antennas $M$ and channel taps $L$.

effect is observed-when the number of users and channel taps are great, the noise is almost circularly symmetric Gaussian and identically distributed around each symbol point for singlecarrier transmission too. This is intuitive, since the precoded transmit signals are the sums of many independent symbols and the receive signals are the sums of many different transmit signals. The in-band distortion therefore gets mixed up and its distribution becomes symmetric and identical around all symbol points, just as is the case in OFDM.

In Figure 6, the variance of the in-band distortion seems to roughly follow the scaling law predicted by [31], which says that the distortion variance relative to the signal power scales as $O(\sqrt{K / M})$. Although the in-band distortion seems to disappear with increasing number of antennas, the amplitude reduction due to clipping does not (in Figure 6, it remains at $-0.2 \mathrm{~dB}$ for all system setups), which was not observed in [31]. However, it can be argued that the clipping power-loss is small and only needs to be considered when the amplifiers are operated close to saturation.

\section{B. Data Rate and Power Consumption}

In this section the power $P_{\text {cons }}$ that the base station amplifiers consume is estimated. Even if the discrete-time constantenvelope precoder outperformed the other precoders in the comparisons in Figure 5, in the end, it is in terms of consumed power the precoders should be compared.

The rate $R_{k}(P, \theta)$ in (43) is a function of the transmit power $P=\eta P_{\text {cons }}$, and therefore a function of the operating point of the power amplifiers, which are parametrized by their efficiency $\eta$. In the case of discrete-time constant-envelope precoding, the rate is also a function of the parameter $\theta=\gamma$. And in the case of regularized zero-forcing, it is a function of the parameter $\theta=\rho$. For a given out-of-band radiation requirement, specified by a maximum ACLR level ACLR $R_{\max }$, the sum-rate of the system is thus given by

$$
R\left(P_{\text {cons }}\right)=\max _{\eta, \theta} \sum_{k=1}^{K} R_{k}\left(\eta P_{\text {cons }}, \theta\right),
$$

where the maximization is over all $\theta \in \mathbb{R}^{+}$and over all operating points $\eta \in\left[0, \eta_{\max }\right]$, where $\eta_{\max }$ is the highest operating point that still results in an ACLR below ACLR $R_{\max }$. If the ACLR is not constrained, $\eta_{\max }$ is taken to be the maximum possible efficiency of the given amplifiers and signal type.

In addition to the conventional linear precoders and the discrete-time constant-envelope precoder, the achievable rate was also evaluated for the PAR-aware precoder with the parameter $\lambda=0.25$ chosen as in [18]. The relation between consumed power and the average sum-rate of the system that is shown in Figure 7 has been obtained by computing (81) for many different user distributions $\left\{\beta_{k}\right\}$ and taking an average. Both the cases (i) when the out-of-band radiation is constrained by requiring the ACLR to be below $-45 \mathrm{~dB}$, which is the ACLR requirement in LTE (Long-Term Evolution) [32], and (ii) when ACLR is not constrained are considered.

It can be seen that maximum-ratio precoding works well for low rate requirements but is limited by interference to below a certain maximum rate. Because the SINR in (48) contains distortion that scales with the radiated power, all precoders have a vertical asymptote, above which the rate cannot be increased. Except for discrete-time constant-envelope precoding, whose curve starts to bend away upwards in the plot for 10 users, this vertical asymptote lies outside the scale and cannot be seen for the other precoders however. Since the array gain $\left|g_{k}\right|^{2}$ is smaller for discrete-time constant-envelope precoding than for zero-forcing and regularized zero-forcing, its vertical asymptote is located at a lower rate than the asymptote of zero-forcing and regularized zero-forcing precoding.

Further, it can be seen that regularized zero-forcing and zero-forcing perform equally well when the number of users is small. Regularized zero-forcing has an advantage over zeroforcing when the number of users is large though, because of its ability to balance the resulting array gain and the amount of interuser interference received by the users. The array gain and interference can also be balanced by discrete-time constant-envelope precoding by the parameter $\gamma$, which is why it continues to perform well in the case with 50 users. The PAR-aware precoder, however, can only trade PAR for higher 

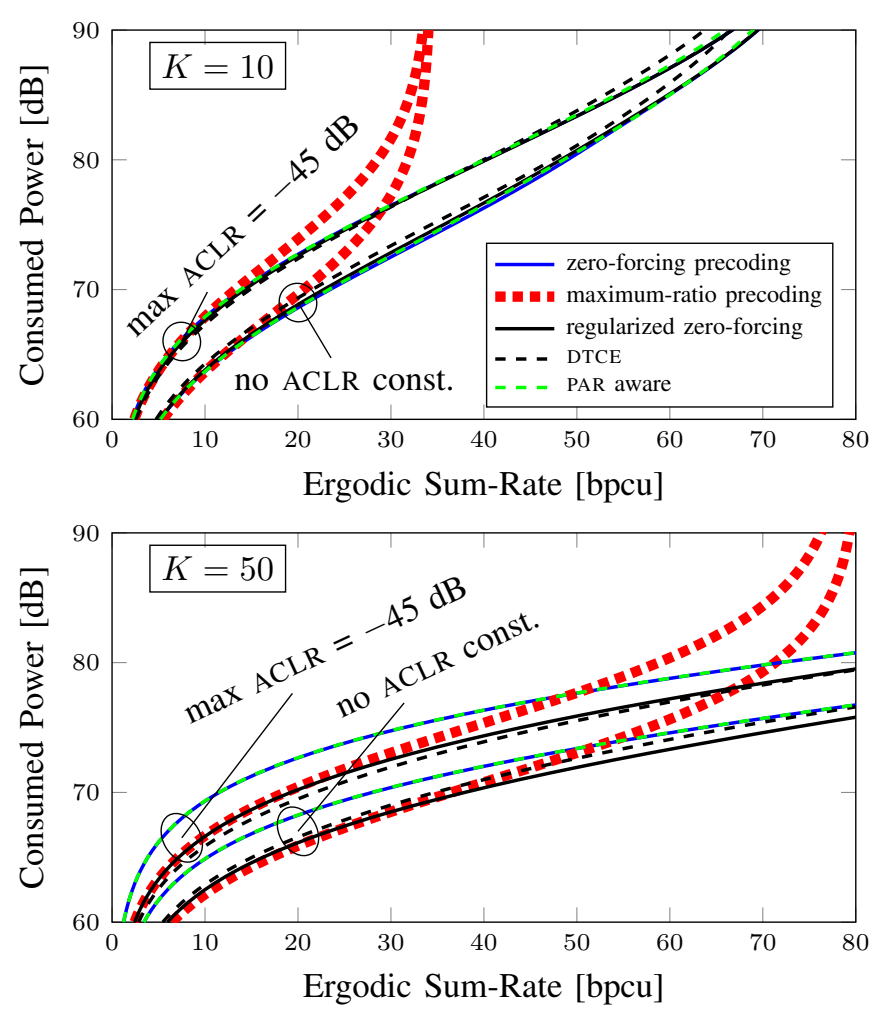

Fig. 7. The estimated consumed power of a base station with $M=100$ antennas required to serve $K=10$ (above) and $K=50$ (below) users with a certain rate over a frequency-selective channel with $L=4$ taps with and without a constraint on the ACLR.

array gain and lower interference by adjusting the parameter $\lambda$. The specific choice of the parameter used here made the precoder behave like zero-forcing; a larger $\lambda$ would lower the PAR of the transmit signals. The PAR-aware precoder, however, cannot balance array gain and interference, which regularized zero-forcing and discrete-time constant-envelope precoding can. Therefore, it performs worse than those precoding techniques when the number of users is large.

The low-PAR precoding technique, discrete-time constantenvelope precoding, seems to consume roughly the same amount of power as the conventional precoding techniques, both when the ACLR is constrained and when it is not, at least for low to medium rate requirements. At very high rates, the optimal linear precoder has an advantage over discretetime constant-envelope precoding-because the array gain of discrete-time constant-envelope precoding is smaller than that of the optimal linear precoder.

The value of $\eta$ that corresponds to the optimal operating point of the amplifiers is shown in Figure 8. When there is no constraint on the out-of-band radiation, it is optimal to operate the amplifiers in saturation, where the power efficiency is high, for low rate requirements. For higher rate requirements, the amplifiers should be backed off to lower the in-band distortion for the conventional precoding techniques. The amplifiers of the discrete-time constant-envelope precoder, however, continue to operate close to saturation also at high rates. When the ACLR is constrained to below $-45 \mathrm{~dB}$, the optimal efficiency of the amplifiers coincides with $\eta_{\max }$ (the

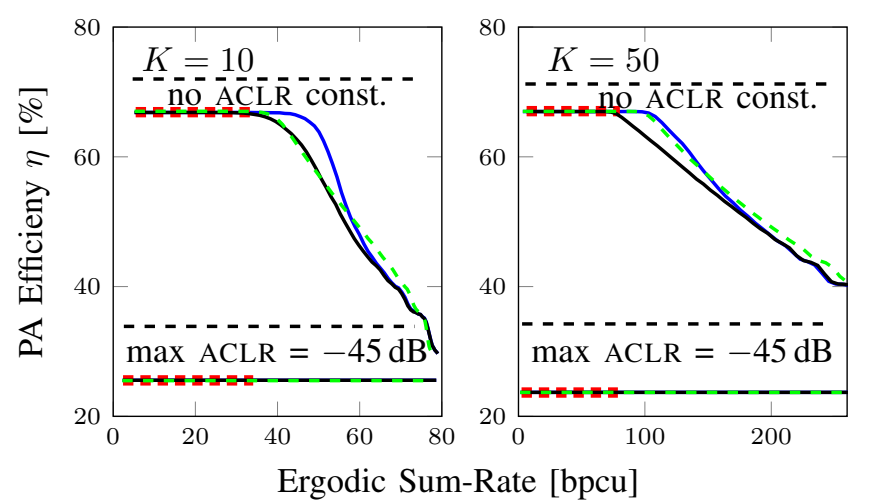

Fig. 8. The power efficiency of the amplifiers at the optimal operating point for different sum-rate requirements. The legend in Figure 7 also applies here.

highest permissible operating point), i.e. $34 \%$ for discretetime constant-envelope precoding and $27 \%$ for maximumratio and zero-forcing precoding, over the whole range of rates investigated, both when serving 10 and 50 users. This corresponds to a backoff of $8 \mathrm{~dB}$ and $11 \mathrm{~dB}$ respectively.

\section{CONCLUSIONS}

We have compared five different multiuser MIMO precoders: maximum-ratio, zero-forcing, regularized zero-forcing, discretetime constant-envelope precoding and PAR-aware precoding. They can be used for single-carrier transmission and for OFDM transmission. The different precoders and transmission methods are summarized in Table II.

In massive MIMO, there is little operational difference between OFDM and single-carrier transmission in terms of complexity, and in terms of PAR. It also turns out that singlecarrier and OFDM transmission have the same performance in terms of data rate. Additionally, massive MIMO allows for time-domain channel inversion to be done with a short filter, for which the number of taps is of the same order of magnitude as the number of channel taps. This makes singlecarrier transmission easy to implement and allows for running precoding with little delays. Since OFDM requires the users to be equipped with an additional FFT (Fast Fourier Transform), this would suggest that single-carrier transmission should be considered in massive MIMO systems.

A massive MIMO system with centralized baseband processing also allows for low-PAR precoding, which increases the power efficiency of the amplifiers but requires more radiated power to compensate for the lowered array gain compared to conventional precoders. For the simplistic power amplifier model used, simulations have shown that the amplifiers of the base station consume the same amount of power when using low-PAR precoding as when using the optimal conventional precoder. Since low-PAR transmit signals are more hardwarefriendly and could enable cheaper and simpler base station designs, this would suggest that a low-PAR precoding technique that also pre-equalizes the channel and suppresses interference (such as discrete-time constant-envelope precoding) should be used in massive MIMO base stations with centralized baseband processing. 
Furthermore, in massive MIMO, simulations have shown that the power efficiency of the amplifiers at the optimal operating point is limited by the out-of-band radiation requirement and that in-band distortion caused by nonlinear amplifiers has two parts: one clipping part that decreases the amplitude of the received signal and one part that can be seen as additive uncorrelated noise, which drowns in the thermal noise in representative cases. The amplitude lost due to clipping is small even when the amplifiers are operated close to saturation.

\section{APPENDIX}

\section{PROOF OF PROPOSITION 1}

We show that single-carrier and OFDM transmission, both with a cyclic prefix added in front of each transmission block, result in the same achievable rate (43). To do that, the effects of the amplifiers are neglected. However, it is reasonable to assume that the in-band distortion caused by the amplifiers affects the two transmission methods in the same way given that the amplitude distributions and PAR of the two transmission methods are the same. The data rate of single-carrier and OFDM are the same if the array gains $\left|g_{k}^{\mathrm{SC}}\right|^{2}=\left|g_{k}^{\mathrm{OFDM}}\right|^{2}$ and interference variances $I_{k}^{\mathrm{SC}}=I_{k}^{\mathrm{OFDM}}$ are the same.

We start by expanding the array gain (36) for single-carrier transmission:

$$
\begin{aligned}
& \left|g_{k}^{\mathrm{SC}}\right|^{2}=\frac{1}{\xi_{k}^{2} \delta_{k}}\left|\mathrm{E}\left[s_{k}^{*}[n] \sum_{m} \sum_{\ell} \hat{h}_{k m}[\ell] u_{m}[n-\ell]\right]\right|^{2} \\
& =\frac{1}{\xi_{k}^{2} \delta_{k}}\left|\mathrm{E}\left[s_{k}^{*}[n] \sum_{m, \ell} \hat{h}_{k m}[\ell] \sum_{k^{\prime}, \ell^{\prime}} w_{m k}\left[\ell^{\prime}\right] s_{k^{\prime}}\left[n-\ell-\ell^{\prime}\right]\right]\right|^{2}
\end{aligned}
$$

Since different symbols are uncorrelated and since they have power $\xi_{k}$, only terms for which $k^{\prime}=k$ and $\ell=-\ell^{\prime}$ will remain, so

$$
\left|g_{k}^{\mathrm{SC}}\right|^{2}=\frac{1}{\delta_{k}}\left|\mathrm{E}\left[\sum_{m} \sum_{\ell} \hat{h}_{k m}[\ell] w_{m k}[-\ell]\right]\right|^{2} .
$$

This is a cyclic convolution evaluated in 0 , which can be computed in the frequency domain followed by an inverse transform:

$$
\left|g_{k}^{\mathrm{SC}}\right|^{2}=\frac{1}{\delta_{k}}\left|\sum_{m} \frac{1}{N} \sum_{n} \mathrm{E}\left[\hat{\tilde{h}}_{k m}[n] \tilde{w}_{m k}[n]\right]\right|^{2} .
$$

If the same precoding technique $f$ has been used for all frequencies, i.e. $f: \tilde{\mathbf{H}}[n] \mapsto \tilde{\mathbf{W}}[n], \forall n$, then all the terms in the inner sum are the same:

$$
\left|g_{k}^{\mathrm{SC}}\right|^{2}=\frac{1}{\delta_{k}}\left|\sum_{m} \mathrm{E}\left[\hat{\tilde{h}}_{k m}[0] \tilde{w}_{m k}[0]\right]\right|^{2},
$$

which is also the array gain $\left|g_{k}^{\text {OFDM }}\right|^{2}$ of OFDM.

We now study the interference (40) for single-carrier transmission, by using (84):

$$
\begin{array}{r}
I_{k}^{\mathrm{SC}}=\mathrm{E}\left[\left|\sum_{m} \sum_{\ell} \hat{h}_{k m}[\ell] u_{m}[n-\ell]-g_{k} s_{k}[n]\right|^{2}\right] \\
=\mathrm{E}\left[\mid \sum_{m} \sum_{\ell} \hat{h}_{k m}[\ell] \sum_{k^{\prime}} \sum_{\ell^{\prime}} w_{m k^{\prime}}\left[\ell^{\prime}\right] s_{k^{\prime}}\left[n-\ell-\ell^{\prime}\right]\right. \\
\left.-\left.s_{k}[n] \mathrm{E}\left[\sum_{m} \sum_{\ell} \hat{h}_{k m}[\ell] w_{m k}[-\ell]\right]\right|^{2}\right]
\end{array}
$$

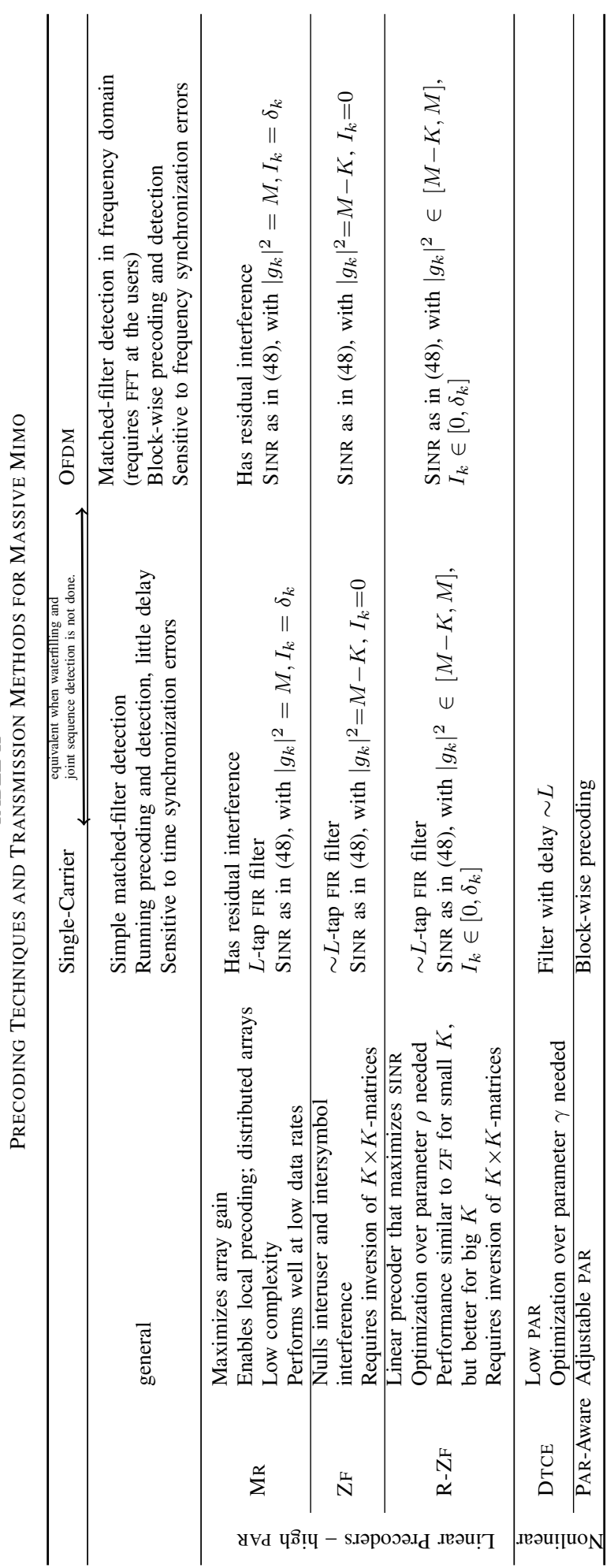




$$
\begin{gathered}
=\mathrm{E}\left[\mid s_{k}[n]\left(\sum_{m, \ell} \hat{h}_{k m}[\ell] w_{m k}[-\ell]-\mathrm{E}\left[\sum_{m, \ell} \hat{h}_{k m}[\ell] w_{m k}[-\ell]\right]\right)\right. \\
\left.+\left.\sum_{\left(k^{\prime}, n^{\prime}\right) \neq(k, 0)} \sum_{k^{\prime}}\left[n^{\prime}\right] \sum_{m} \sum_{\ell} \hat{h}_{k m}[\ell] w_{m k^{\prime}}\left[n^{\prime}-\ell\right]\right|^{2}\right]
\end{gathered}
$$

Since different symbols are uncorrelated and since they have power $\xi_{k}$, the square is expanded into the following.

$$
\begin{aligned}
I_{k}^{\mathrm{SC}}=\sum_{k^{\prime}} \sum_{n^{\prime}} \xi_{k^{\prime}} & \mathrm{E}\left[\left|\sum_{m} \sum_{\ell} \hat{h}_{k m}[\ell] w_{m k^{\prime}}\left[n^{\prime}-\ell\right]\right|^{2}\right] \\
& -\xi_{k}\left|\mathrm{E}\left[\sum_{m} \sum_{\ell} \hat{h}_{k m}[\ell] w_{m k}[-\ell]\right]\right|^{2}
\end{aligned}
$$

The two terms are cyclic convolutions in $n^{\prime}$ and 0 respectively and can be computed in the frequency domain. Along the same line of reasoning as in (86), the interference variance is given by $I_{k}^{\mathrm{SC}}=$

$$
\sum_{k^{\prime}} \xi_{k^{\prime}} \mathrm{E}\left[\left|\sum_{m} \hat{\tilde{h}}_{k m}[0] \tilde{w}_{m k^{\prime}}[0]\right|^{2}\right]-\xi_{k}\left|\mathrm{E}\left[\sum_{m} \hat{\tilde{h}}_{k m}[0] \tilde{w}_{m k}[0]\right]\right|^{2},
$$

which is precisely the interference variance $I_{k}^{\text {OFDM }}$ of OFDM at tone 0 , or at any other tone.

That the rate (43) is the same for single-carrier and OFDM transmission was proven, in a different way, for the special case maximum-ratio precoding in [24].

\section{REFERENCES}

[1] C. Mollén, E. G. Larsson, and T. Eriksson, "On the impact of PA-induced in-band distortion in massive MIMO," The Proceedings of the European Wireless Conference, May 2014.

[2] T. L. Marzetta, "Noncooperative cellular wireless with unlimited numbers of base station antennas," IEEE Transactions on Wireless Communications, vol. 9, no. 11, pp. 3590-3600, Oct. 2010.

[3] E. G. Larsson, O. Edfors, F. Tufvesson, and T. L. Marzetta, "Massive MIMO for next generation wireless systems," IEEE Communications Magazine, vol. 52, no. 2, pp. 186-195, Feb. 2014.

[4] H. Q. Ngo, E. G. Larsson, and T. L. Marzetta, "Energy and spectral efficiency of very large multiuser MIMO systems," IEEE Transactions on Communications, vol. 61, no. 4, pp. 1436-1449, Feb. 2013.

[5] H. Yang and T. L. Marzetta, "Performance of conjugate and zero-forcing beamforming in large-scale antenna systems," IEEE Journal on Selected Areas in Communications, vol. 31, no. 2, pp. 172-179, Feb. 2013.

[6] J. Hoydis, K. Hosseini, S. t. Brink, and M. Debbah, "Making smart use of excess antennas: Massive MIMO, small cells, and TDD," Bell Labs Technical Journal, vol. 18, no. 2, pp. 5-21, Sep. 2013.

[7] G. Caire and S. Shamai, "On the achievable throughput of a multiantenna Gaussian broadcast channel," IEEE Transactions on Information Theory, vol. 49, no. 7, pp. 1691-1706, Jul. 2003.

[8] A. Goldsmith, S. A. Jafar, N. Jindal, and S. Vishwanath, "Capacity limits of MIMO channels," IEEE Journal on Selected Areas in Communications, vol. 21, no. 5, pp. 684-702, Jun. 2003.

[9] M. Joham, W. Utschick, and J. A. Nossek, "Linear transmit processing in MIMO communications systems," IEEE Transactions on Signal Processing, vol. 53, no. 8, pp. 2700-2712, Aug. 2005.

[10] E. Björnson, M. Bengtsson, and B. Ottersten, "Optimal multiuser transmit beamforming: A difficult problem with a simple solution structure," IEEE Signal Processing Magazine, vol. 31, no. 4, pp. 142-148, Jul. 2014.

[11] M. Vu, "MISO capacity with per-antenna power constraint," IEEE Transactions on Communications, vol. 59, no. 5, pp. 1268-1274, Mar. 2011.

[12] — "MIMO capacity with per-antenna power constraint," in Proc. IEEE Global Telecommunications Conference, Dec. 2011, pp. 1-5.

[13] W. Yu and T. Lan, "Transmitter optimization for the multi-antenna downlink with per-antenna power constraints," IEEE Transactions on Signal Processing, vol. 55, no. 6, pp. 2646-2660, Jun. 2007.
[14] H. Hemesi, A. Abdipour, and A. Mohammadi, "Analytical modeling of MIMO-OFDM system in the presence of nonlinear power amplifier with memory," IEEE Transactions on Communications, vol. 61, no. 1, pp. 155-163, Nov. 2013.

[15] H. Ochiai, "An analysis of band-limited communication systems from amplifier efficiency and distortion perspective," IEEE Transactions on Communications, vol. 61, no. 4, pp. 1460-1472, Feb. 2013.

[16] S. Mohammed and E. G. Larsson, "Constant-envelope multi-user precoding for frequency-selective massive MIMO systems," IEEE Wireless Communications Letters, vol. 2, no. 5, pp. 547-550, Oct. 2013.

[17] — , "Per-antenna constant envelope precoding for large multi-user MIMO systems," IEEE Transactions on Communications, vol. 61, no. 3, pp. 1059-1071, Mar. 2013.

[18] C. Studer and E. G. Larsson, "PAR-aware large-scale multi-user MIMOOFDM downlink," IEEE Journal on Selected Areas in Communications, vol. 31, no. 2, pp. 303-313, Feb. 2013.

[19] E. Björnson, J. Hoydis, M. Kountouris, and M. Debbah, "Massive MIMO systems with non-ideal hardware: Energy efficiency, estimation, and capacity limits," IEEE Transactions on Information Theory, vol. 60 no. 11, pp. 7112-7139, Nov. 2014

[20] E. Costa, M. Midrio, and S. Pupolin, "Impact of amplifier nonlinearities on OFDM transmission system performance," IEEE Communications Letters, vol. 3, no. 2, pp. 37-39, Feb. 1999.

[21] J. Qi and S. Aïssa, "Analysis and compensation of power amplifier nonlinearity in MIMO transmit diversity systems," IEEE Transactions on Vehicular Technology, vol. 59, no. 6, pp. 2921-2931, May 2010.

[22] M. Médard, "The effect upon channel capacity in wireless communications of perfect and imperfect knowledge of the channel," IEEE Transactions on Information Theory, vol. 46, no. 3, pp. 933-946, May 2000.

[23] B. Hassibi and B. M. Hochwald, "How much training is needed in multiple-antenna wireless links?" IEEE Transactions on Information Theory, vol. 49, no. 4, pp. 951-963, Apr. 2003.

[24] A. Pitarokoilis, S. K. Mohammed, and E. G. Larsson, "On the optimality of single-carrier transmission in large-scale antenna systems," IEEE Wireless Communications Letters, vol. 1, no. 4, pp. 276-279, Apr. 2012

[25] A. M. Tulino and S. Verdú, Random Matrix Theory and Wireless Communications. Now Publishers Inc., 2004, vol. 1.

[26] L. Sanguinetti, E. Björnson, M. Debbah, and A. L. Moustakas, "Optimal linear precoding in multi-user MIMO systems: A large system analysis," in The Proceedings of the IEEE Global Communications Conference, Dec. 2014, pp. 3922-3927.

[27] S. Mohammed and E. G. Larsson, "Single-user beamforming in largescale MISO systems with per-antenna constant-envelope constraints: The doughnut channel," IEEE Transactions on Wireless Communications, vol. 11, no. 11, pp. 3992-4005, Sep. 2012.

[28] H. Yang and T. L. Marzetta, "A macro cellular wireless network with uniformly high user throughputs," in The Proceedings of the Vehicular Technology Conference, Sep. 2014, pp. 1-5.

[29] N. Benvenuto, R. Dinis, D. Falconer, and S. Tomasin, "Single carrier modulation with nonlinear frequency domain equalization: An idea whose time has come-again," Proceedings of the IEEE, vol. 98, no. 1, pp 69-96, 2010.

[30] F. H. Raab, P. Asbeck, S. Cripps, P. B. Kenington, Z. B. Popovic, N. Pothecary, J. F. Sevic, and N. O. Sokal, "Power amplifiers and transmitters for RF and microwave," IEEE Transactions on Microwave Theory and Techniques, vol. 50, no. 3, pp. 814-826, Mar. 2002.

[31] E. Björnson, M. Matthaiou, and M. Debbah, "Massive MIMO with arbitrary non-ideal arrays: Hardware scaling laws and circuit-aware design," ArXiv E-Print, Sep. 2014, arXiv:1409.0875 [cs.IT].

[32] 3GPP TS36.141 3rd Generation Partnership Project; Technical Specification Group Radio Access Network; Evolved Universal Terrestrial Radio Access (E-UTRA); Base Station (BS) Conformance Testing (Release 10), 3GPP Std., Rev. V10.2.0, 2011. 


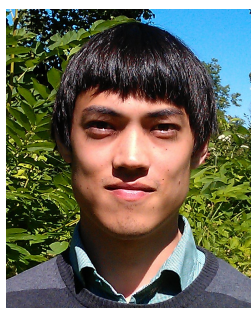

as a Fulbright Scholar.
Christopher Mollén received his M.Sc. degree from Linköping University, Sweden, in 2013, where he is currently pursuing his the Ph.D. degree with the Department of Electrical Engineering, Division for Communication Systems. His research interest is lowcomplexity hardware implementations of massive MIMO base stations, including low-PAR precoding, one-bit ADCs, and nonlinear amplifiers. Prior to his Ph.D. studies, he worked as an Intern at Ericsson in Kista, Sweden, and in Shanghai, China. From 2015 to 2016, he visited the University of Texas at Austin

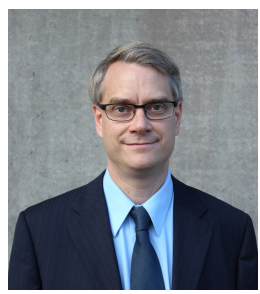

Erik G. Larsson received his Ph.D. degree from Uppsala University, Sweden, in 2002. Since 2007 he is Professor in the Department of Electrical Engineering (ISY) at Linköping University (LiU) in Linköping, Sweden. He has previously been Associate Professor (Docent) at the Royal Institute of Technology (KTH) in Stockholm, Sweden, and an Assistant Professor with the University of Florida and the George Washington University, USA. In the spring of 2015 he was a Visiting Fellow at Princeton University, USA, for four months.

His main professional interests are within the areas of wireless communications and signal processing. He has authored some 120 journal papers on these topics, co-authored the textbook entitled Space-Time Block Coding for Wireless Communications (Cambridge Univ. Press, 2003) and holds 15 issued and several pending patents on wireless technology.

He received the IEEE Signal Processing Magazine Best Column Award twice, in 2012 and 2014, and the IEEE ComSoc Stephen O. Rice Prize in Communications Theory in 2015. He served as Associate Editor for several major journals, including the IEEE Transactions on Communications (2010-2014) and IEEE Transactions on Signal Processing (2006-2010). He serves as chair of the IEEE Signal Processing Society SPCOM technical committee in 2015-2016 and he served as chair of the steering committee for the IEEE Wireless Communications Letters from 2014 to 2015. He was the General Chair of the Asilomar Conference on Signals, Systems and Computers in 2015, and Technical Chair in 2012. He is a Fellow of the IEEE.

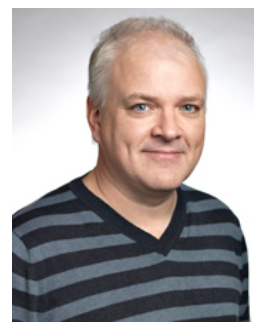

Thomas Eriksson received the Ph.D. degree in information theory from Chalmers University of Technology, Gothenburg, Sweden, in 1996. From 1990 to 1996, he was with Chalmers University of Technology. In 1997 and 1998, he was with AT\&T Labs-Research, Murray Hill, NJ, USA. In 1998 and 1999, he was with Ericsson Radio Systems AB, Kista, Sweden. Since 1999, he has been with Chalmers University of Technology, where he is currently a Professor of communication systems. Furthermore, he was a Guest Professor with Yonsei University, South Korea, from 2003 to 2004. He has authored or co-authored over 200 journal and conference papers, and holds eight patents.

$\mathrm{He}$ is leading the research efforts on hardware-constrained communications with Chalmers University of Technology.

His research interests include communication, data compression, and modeling and compensation of nonideal hardware components (e.g., amplifiers, oscillators, and modulators in communication transmitters and receivers, including massive MIMO). He is currently the Vice Head of the Department of Signals and Systems with the Chalmers University of Technology, where he is responsible for undergraduate and master's education. 